\title{
A Protective Mattress Development for Patients with Peripheral Vascular Disease*
}

\author{
Fumiko FURUKAWA**, Noriyoshi TANAKA***, Mitsunori MIYASHITA****, \\ Haruko SHIKIMORI ** and Hiroaki KANAZAWA**
}

\section{Introduction}

Blood flow to the legs of patients with peripheral vascular disease (PVD) is often insufficient ${ }^{1,2}$. Microcirculation in the legs of these patients is easily affected by interface pressure $^{3)}$ and shear force ${ }^{4)}$, which result in pressure-related skin damage ${ }^{5)}$. One area where pressure ulcers of the lower legs commonly develop is the heel ${ }^{5,6}$. In addition, ulcers may develop anywhere on the calf and foot in patients with combined arterial and venous insufficiency ${ }^{1}$, although the calves are not commonly exposed to pressure and shear force. However, the effect of shear force arising from the larger muscles on the calves should be considered ${ }^{7)}$. The calves are particularly affected when patients with PVD spend their time resting in bed to avoid aggravation of pain, numbness, or cold feelings in the lower legs caused by excessive use from walking and similar activities.

Capillary blood flow is stopped by a peak pressure above $32 \mathrm{mmHg}^{8)}$. Indirectly measured peak pressure over 40 to $50 \mathrm{mmHg}$ may disturb the blood flow of the microcirculation ${ }^{9)}$, and this pressure, acting as a physical stressor on capillary vessels, may activate the sympathetic nervous system, causing vascular constriction ${ }^{10)}$. Simultaneously, compensatory mechanisms are initiated so that the microcirculation supplies blood against the peak pressure $^{11,12)}$. Permeability of the capillary wall may increase

* Received January 25, 2012 ; accepted May 23, 2012

** School of Nursing, University of Shizuoka

*** Department of Nursing, Tokai University Hospital

****Department of Palliative Nursing, Health Science, Tohoku University Graduate School of Medicine if the microcirculation is blocked by continued pressure, leading to an inflammatory response, such as edema, around the area of peak pressure ${ }^{13)}$.

Shear force that acts as a physical stressor causes capillary displacement and strain ${ }^{4)}$. Such mechanical changes in the vessels lead to further vasoconstriction of capillary vessels. Shear force also directly causes breakdown of the skin ${ }^{14)}$. Thus, for patients with PVD, two items are critical : (1) redistributing the peak pressure with respect to both the heels and the calves by expanding the contact area and (2) reducing the external shear force by controlling the sliding motion of the leg.

Many pressure-relieving devices consisting of foam, gel, or air have been evaluated ${ }^{3,15,16)}$. However, there are few devices that fit and sustain the morphological shape of the calves and heels in patients with PVD to allow reduction of the peak pressure and shear force. Because PVD is a significant risk factor for pressure-related skin damage $\mathrm{e}^{2,5)}$ and the development of ischemic and venous skin ulcers, as well as interferes with the healing process for these ulcers ${ }^{17)}$, the aim of this experimental laboratory study was to develop and test a protective mattress for calves and heels in patients with PVD using a dummy human leg as a model.

\section{Methods}

\section{2-1. Theoretical framework for design of a protective mattress}

Interface pressure and shear force are major risk factors for skin and soft tissue damage ${ }^{7)}$, and these factors should be controlled to minimize cessation of the microcirculation, 
especially for patients with PVD who have insufficient blood flow to the legs. To reduce peak pressure, the contact area of the calves and heels should be expanded by sinking the calves and heels without allowing them to sink to the bottom of the mattress. To minimize shear force, the calves and heels should also be sunk vertically downward to reduce the sliding motion of the legs on the mattress. The theoretical framework to support the design of the protective mattress for the present study thus was that "the calves and heels are sunk vertically downward while being gently held, and then stabilized firmly without sinking to the mattress bottom.”

\section{2-2. Study design}

Due to ethical considerations arising from frequent measurements involving human subjects, we designed a dummy human leg by taking a leg from a full body flexible manikin made of polystyrol foam (Suncreate, Ooita, Japan), and subsequently used it in the laboratory experimental study for developing and testing the protective mattresses.

A leg was used because it was assumed to be representative of a full body. Based on a study reported by Se Jin Park, et al. ${ }^{18)}$, mass proportions of each body segment can be represented as a percentage of the weight of the whole body. Particularly, the rationale for using only a leg in our study came from four measurements : the peak pressure and contacted area of both the heel and the calf in the supine position, without elevating the upper body. In this case, pressure created by the weight of the leg should be relatively unaffected by the pressure arising from the weight of the buttocks or upper body, so that any differences in measurement between using only the leg and the leg with the full body should be small. Thus, the values obtained from measuring a leg only are considered to be applicable for estimating those obtained from measuring the leg with the weight of the whole body.

The primary factors measured were peak pressure and the distance of the sliding motion of the weight representing shear force. We hypothesized that peek pressure is below $50 \mathrm{mmHg}$, and the distance of the sliding motion representing shear force is nearly equal to $1 \mathrm{~mm}$ or zero.

\section{2-3. Making a dummy human leg}

A dummy human leg representing the medium-sized
Japanese women, aged 20 years and older was designed : flexion and extension of the knee joint were possible, but the ankle joint was fixed. To generate human-like skin and subcutaneous tissue, the lower leg was wrapped with an 8mm-thick layer of soft silicon gel and baked at $60^{\circ} \mathrm{C}$ for $8 \mathrm{~h}$ in an oven. A similar soft layer was also applied to the heel. Because the weight of the dummy leg was only $1.6 \mathrm{~kg}$, additional weight $(9.5 \mathrm{~kg})$ was added using sandbags of $0.5,3.0$, and $6.0 \mathrm{~kg}$ on the foot, shin, and thigh, respectively. This additional weight made the leg weigh approximately the same as that of a typical $60-\mathrm{kg}$ female leg $(11.1 \mathrm{~kg})$, representing approximately $18.7 \%$ of total body weight.

\section{2-4. Preparing the protective mattresses}

The protective mattress was created based on the theoretical framework described above. To fully expand the contact area by supporting the morphological shape of the calves and heels, soft, thick materials are required. On the other hand, characteristics of materials that provide pressure-relief may allow the legs to slide in various directions if they do not firmly maintain the weight of the legs, resulting in the generation of shear force at the skinmaterial interface. To minimize the sliding motion of the legs on the mattress, the material requires some firmness, which also prevents the legs from sinking to the bottom of the mattress.

Clearly, adequate thickness, softness, and firmness of the materials are critical for the legs of patients with PVD. Therefore, materials made from a 3-dimensional knit fabric (Fusion $^{\circledR}$, Asahikasei, Osaka, Japan) sandwiched between two layers of fabric with different textures were selected. Seven patterns of fabric were used in the present study (Tab. 1).

Tab. 1 Characteristics of the seven Fusion ${ }^{\circledR}$ materials.

\begin{tabular}{|c|c|c|c|c|c|}
\hline \multirow[t]{2}{*}{ Material } & \multirow{2}{*}{$\begin{array}{c}\text { Density } \\
(\mathrm{g} / \mathrm{m})\end{array}$} & \multirow{2}{*}{$\begin{array}{l}\text { Thickness } \\
(\mathrm{mm})\end{array}$} & \multicolumn{3}{|c|}{ Composition } \\
\hline & & & Front & Middle & Back \\
\hline A & 520 & 3.1 & Coated T & $\mathrm{N}$ & ET \\
\hline B & 400 & 2.4 & ET & $\mathrm{N}$ & ET \\
\hline $\mathrm{C}$ & 430 & 10.0 & ET & S & ET \\
\hline $\mathrm{D}$ & 1140 & 8.0 & ET & S & ET \\
\hline $\mathrm{E}$ & 670 & 4.0 & ET & $\mathrm{N}$ & ET \\
\hline $\mathrm{F}$ & 480 & 3.5 & Coated T & $\mathrm{N}$ & ET \\
\hline G & 520 & 3.8 & ET & $\mathrm{N}$ & ET \\
\hline
\end{tabular}


These seven fabrics were combined in different thicknesses and softness using three strategies : 1) stratifying materials with different thicknesses and softness ; 2) making a double-fold of a single layer of each material ; and 3) stacking each double-fold by rotating

Tab. 2 Characteristics created by strategies to actualize the theoretical framework for the protective mattress.

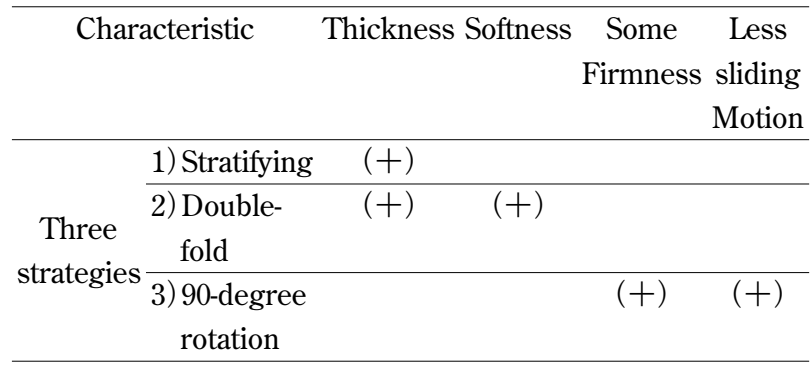

$(+)$ indicates the presence of each characteristics created by strategy 1), 2), or 3 ).

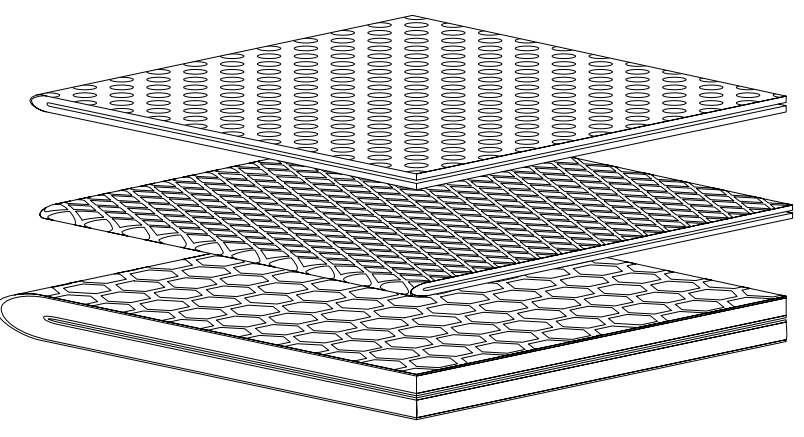

Fig. 1 A protective mattress with a 90-degree-rotated layer. This figure shows the application of three strategies : 1) stratifying materials with different thicknesses and softnesses ; 2) making a double-fold of a single layer of each material ; and 3) rotating each double-fold by 90 degrees.

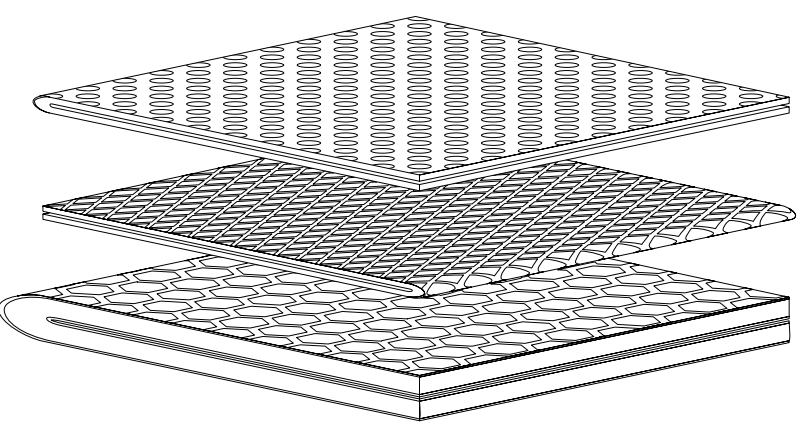

Fig. 2 A protective mattress without a 90-degree-rotated layer: This figure shows the application of two strategies : 1) stratifying materials with different thicknesses and softnesses ; and 2) making a doublefold of a single layer of each material. Note that each double-fold is not rotated by 90 -degrees in this figure. them by 90 degrees. By combining three or four of the seven patterns of materials using the three strategies, protective mattresses were constructed, and functional characteristics were presented in Tab. 2.

An example of a protective mattress with 90-degree rotation designed using strategies 1),2), and 3) is shown in Fig. 1, and an example of a protective mattress with nondegree rotation, that is, using strategies 1) and 2) only, is shown in Fig. 2.

The height of the protective mattress for the leg was less than $5.0 \mathrm{~cm}$, with the legs elevated approximately 10 degrees. This height for leg elevation was selected for safety reasons : 10-degree leg elevation in the less than 30degree head-up position results in no significant differences in the mean interface pressure at the sacrum ${ }^{19)}$. Moreover, because arterial and venous insufficiency usually co-exist in patients with PVD, the legs of these patients should be in the appropriate position, that is, neither too high nor too low, to minimize problems arising from support pressure and capillary perfusion pressure ${ }^{5)}$.

\section{2-5. Measurement for peak pressure and the contact area}

Xsensor3 (ver. 5 ; Xsensor, Calgary, Canada), an interface pressure mapping device that records the distribution of pressure over the body, was used to measure peak pressure and contact area (Fig. 3). This mapping device consists of a mat made of a square array of 6912 pressure-sensitive transducers spaced $12.5 \mathrm{~mm}$ apart encased in a woven nylon envelope $(61 \mathrm{~cm} \times 183 \mathrm{~cm})$, with drape properties similar to a bed sheet. The $12.5 \mathrm{~mm}^{2}$ individual pressure sensing cells are capable of measuring

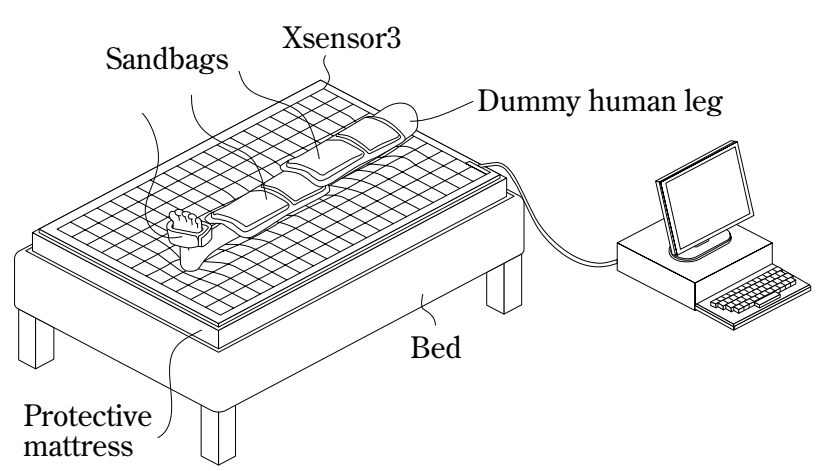

Fig. 3 Measurement for the peak pressure and the contacted area of the heel and the calf of the leg. 
the pressures across a wide range $(5 \sim 200 \mathrm{mmHg})$ and, collectively, can provide the pressure distribution of objects placed on the mat. The Xsensor3 was connected to a computer, and data were recorded using software built for this application (ver. 5 ; Xsensor, Calgary, Canada). Calibration of the mapping system was performed by a technical assistant as per the manufacturer's instructions prior to the testing day. Each recording was taken after 3 minutes had passed to minimize transient creep behavior of the mattress on loading, and data during the next 15 to 20 seconds, based on one frame per second, were used. The accuracy of measurements of peak pressure and contact area using the Xsensor3 has been confirmed in both a laboratory experimental study and human subjects ${ }^{20,21)}$.

\section{2-6. Measurement for deviation in sliding distance}

The degree of sliding motion that appeared when a weight was placed on the protective mattresses was measured. It is important to measure the magnitude of this sliding motion because it creates shear force that may indirectly cause capillary displacement or strain and directly damage the skin. Although it is difficult to reliably and noninvasively measure shear force, and the clinical relevance of the degree of shear force is unknown ${ }^{21)}$, we attempted to measure it by measuring sliding motion. A calibration weight of $1.0 \mathrm{~kg}$ (Mettler, Greifensee, Switzerland) and a laser pointer were used for this part of the study (Fig. 4).

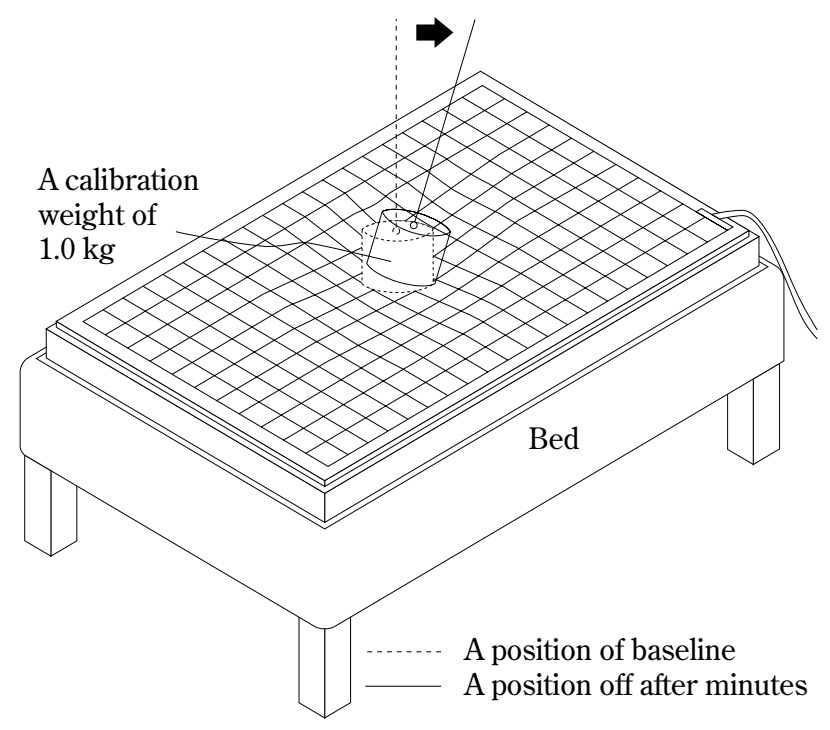

Fig. 4 Measurement for deviation in sliding distance.
First, the center of the round surface of the calibration weight was marked using an oil-based pen. Second, the weight was manually placed exactly below the spot of the laser beam, and laser light fixed $30 \mathrm{~cm}$ above the mattress was directed to the point at the center of the calibration weight placed on the mattress. Then, the calibration weight was slowly released. It gradually sank while being allowed to slide in any direction. After waiting 1 minute for the inclination of the calibration weight to stabilize, the distance between the center previously marked and the spot at the center of the calibration weight identified by the laser light was measured 30 times every 15 seconds using a certified stainless steel metric measure (length : $600 \mathrm{~mm}$; width : $30 \mathrm{~mm}$, with 1/2-mm intervals) (Shinwa, Niigata, Japan). A $1.0 \mathrm{~kg}$ of calibration weight was selected because it is nearly equal to the foot's weight of the medium-sized Japanese women on the heel at resting in bed (one area where pressure ulcers of the lower legs commonly develop).

\section{2-7. Procedures}

The testing procedure was carried out in a temperaturecontrolled laboratory $\left(24\right.$ to $26^{\circ} \mathrm{C}$, with approximately $60 \%$ humidity) built for this study. A hospital bed (Paramount Bed, Tokyo, Japan) was prepared with a 7.5-cm-high polyurethane mattress (Preglar, Tokyo, Japan) and a 7mm-thick cotton mattress pad covered with a $100 \%$ cotton sheet. For baseline data of the peak pressure and the contact area, the Xsensor3 mat was spread on the bed, and then the dummy human leg placed on the Xsensor3 mat. Baseline data were measured three times under identical conditions, and the second set of data was used as the baseline data for the present study. Three or four materials were first combined by applying strategies 1) (stratifying layers) and 2) (each layer double-folded), and more than 100 fabric combinations of the mattress were experimentally measured to generate better thickness and softness and lower peak pressure with the expanded contact area. Of the more than 100 combinations, 10 were finally chosen based on the values of the peak pressure and thickness and softness of the mattress. Softness was confirmed using the textural sense of three researchers.

Second, these 10 combinations were measured by applying strategy 3) (90-degree rotation) to test the peak pressure and the contact area. Each combined mattress 
was placed again on the bed, the Xsensor3 was spread on the mattress, and the dummy human leg was then placed on the Xsensor3. Each measurement was taken under identical conditions. Finally, the sliding distance, representing the shear force, was measured using the method described in "Degree of sliding motion." Mattresses that indicated better outcomes, that is, lower peak pressure on the heel and calf than baseline values and thickness above $34.0 \mathrm{~mm}$, were selected. Visual inspection was used to confirm that the calf and heel did not sink to the bottom of the mattress.

\section{2-8. Data analysis}

Each recording for peak pressure and contact area for each combination was exported to spreadsheet software (Microsoft Excel, Microsoft Asian Ltd., Japan), and values for each combination were calculated based on the recording method described in "Measurement Tools." The mean sliding distance of the mattresses with 90 - and nondegree rotation were tested by Mann-Whitney $\mathrm{U}$ test due to non-normal distribution of the data. To test for significance of the sliding distance among three mattresses with 90degree rotation, Kruskal-Wallis test was applied. The analyses were preformed in the SPSS package (SPSS, IBM company, Version 18.0, Tokyo, Japan). The level for significance was $\mathrm{p}<.05$.

\section{Results}

Baseline data for peak pressure and contact area were $152.8 \mathrm{mmHg}$ and $124.2 \mathrm{~cm}^{2}$, respectively, for the calf, and $36.1 \mathrm{mmHg}$ and $9.7 \mathrm{~cm}^{2}$, respectively, for the heel. The peak pressures and contact areas of the mattresses using the three strategies were shown in Tab. 3.

The peak pressures on the calf with 90-degree rotation for all combinations were approximately one fifth of the baseline peak pressures. Of all the combinations, AFCD, $\mathrm{ABCD}$, and $\mathrm{ACE}$ had better outcomes in peak pressure,

Tab. 4 Significances in sliding distance of the mattresses with non-degree and 90-degree rotation for combinations $\mathrm{AFCD}, \mathrm{ABCD}$, and ACE.

\begin{tabular}{cccc}
\hline Combinations & $\begin{array}{c}\text { Mean } \pm \mathrm{SD} \\
(\mathrm{mm})\end{array}$ & $\begin{array}{c}95 \% \mathrm{CI} \dagger \\
(\mathrm{mm})\end{array}$ & P value* \\
\cline { 2 - 4 } AFCD & & & \\
Non-degree & $4.52 \pm 1.67$ & $3.89-5.14$ & $\mathrm{P}<.01$ \\
90-degree & $1.45 \pm 0.62$ & $1.22-1.68$ & \\
ABCD & & & \\
Non-degree & $5.08 \pm 0.92$ & $4.47-5.43$ & $\mathrm{P}<.01$ \\
90-degree & $2.08 \pm 0.44$ & $1.92-2.25$ & \\
ACE & & & \\
Non-degree & $4.57 \pm 1.04$ & $4.18-4.96$ & $\mathrm{P}<.01$ \\
90-degree & $3.30 \pm 0.62$ & $3.07-3.53$ & \\
\hline *: Mann-Whitney U test $\dagger: 95$ Percent Confidence Interval
\end{tabular}

Tab. 3 Peak pressure and the contact area achieved with different combinations of the seven materials.

\begin{tabular}{|c|c|c|c|c|c|c|c|c|c|c|}
\hline \multirow{2}{*}{$\begin{array}{c}\text { Combination } \\
\text { (Double-fold } \\
\text { 90-degree rotation) } \\
\text { (Thickness in } \mathrm{mm} \text { ) }\end{array}$} & & \multicolumn{2}{|c|}{ Calibration } & \multicolumn{2}{|c|}{ Heel } & & \multicolumn{2}{|c|}{ Calf } & & \\
\hline & & $\begin{array}{c}\text { Contact } \\
\text { Area } \\
\left(\mathrm{cm}^{2}\right)\end{array}$ & $\begin{array}{c}\text { Peak } \\
\text { pressure } \\
(\mathrm{mmHg})\end{array}$ & $\begin{array}{c}\text { Contact } \\
\text { area } \\
\left(\mathrm{cm}^{2}\right)\end{array}$ & $\begin{array}{c}\text { Peak } \\
\text { pressure } \\
(\mathrm{mmHg})\end{array}$ & & $\begin{array}{c}\text { Contact } \\
\text { Area } \\
\left(\mathrm{cm}^{2}\right)\end{array}$ & $\begin{array}{c}\text { Peak } \\
\text { pressure } \\
(\mathrm{mmHg})\end{array}$ & & \\
\hline Baseline & & 24.2 & 45.0 & 9.7 & 36.1 & & 124.2 & 152.8 & & \\
\hline $\operatorname{AFCD}(49.2 \mathrm{~mm})$ & * & 22.6 & 28.6 & 14.5 & 33.4 & $\dagger$ & 145.2 & 42.2 & 末 & $\S$ \\
\hline $\mathrm{AACD}(48.4 \mathrm{~mm})$ & $*$ & 22.6 & 36.4 & 17.7 & 36.1 & & 141.9 & 37.6 & $\ddagger$ & \\
\hline $\mathbf{A B C D}(47.0 \mathrm{~mm})$ & $*$ & 24.2 & 28.1 & 16.1 & 35.9 & $\dagger$ & 137.1 & 43.4 & $\neq$ & $\S$ \\
\hline $\mathrm{ABDC}(47.0 \mathrm{~mm})$ & $*$ & 22.6 & 27.2 & 16.1 & 36.4 & & 132.3 & 45.1 & $\ddagger$ & \\
\hline $\operatorname{ACC}(46.2 \mathrm{~mm})$ & $*$ & 22.6 & 26.5 & 22.6 & 38.3 & & 138.7 & 38.6 & $\ddagger$ & \\
\hline $\mathbf{A C E}(34.2 \mathrm{~mm})$ & $*$ & 22.6 & 25.4 & 14.5 & 33.3 & $\dagger$ & 135.5 & 43.0 & $\ddagger$ & $\S$ \\
\hline $\operatorname{AGC}(33.8 \mathrm{~mm})$ & & 25.8 & 26.5 & 21.0 & 35.9 & $\dagger$ & 132.3 & 40.9 & $\ddagger$ & \\
\hline $\operatorname{AFC}(33.2 \mathrm{~mm})$ & & 21.0 & 30.6 & 22.6 & 37.1 & & 138.7 & 39.5 & $\ddagger$ & \\
\hline $\mathrm{AAC}(32.4 \mathrm{~mm})$ & & 24.2 & 31.3 & 17.7 & 35.4 & $\dagger$ & 135.5 & 41.4 & $\ddagger$ & \\
\hline $\mathrm{ABC}(31.0 \mathrm{~mm})$ & & 24.2 & 26.5 & 16.1 & 35.3 & $\dagger$ & 138.7 & 42.7 & $\neq$ & \\
\hline
\end{tabular}

See Tab. 1 for the composition of a single layer of fabrics A, B, C, D, E, F, and G.

$*$ : Thickness $>34.2 \mathrm{~mm} ; \dagger$ : Peak pressure of the heel $<$ baseline value $; \ddagger$ : Peak pressure of the calf $<$ baseline value ;

$\S:$ The conditions of $*, \dagger$, and $\ddagger$ were met. 
contact area, and thickness

The mean sliding distance using these particular combinations between 90- and non-degree rotation were shown in Tab. 4. Of the three combinations, the weight on AFCD exhibited the smallest sliding distance $\left(\chi^{2}=61.61\right.$, $\mathrm{df}=2, \mathrm{p}<.01)$.

\section{Discussion}

Protective mattresses were created for legs of patients with PVD. Stratified, double-folded, and 90-degree-rotated layers showed significant improvements in peak pressure, contact area, and sliding motion. In particular, the peak pressure for the calf in all combinations was lower than the pressure indicated by Sugama et $a l .^{9)}$ to stop capillary blood flow. The distance of the sliding motion representing shear force was very small. Thus, the hypotheses were confirmed. These findings of this study may indicate effectiveness in preventing severe blockage of the microcirculation of the legs arising from interface pressure and shear force. Because the significant mattresses with primary outcomes attained were identified by the three strategies created by the theoretical framework, the unique features of such a mattress are discussed here.

First, mattress thickness was an important factor for expanding the contact area. As we expected, stratified layers with different thicknesses provided enough thickness to maintain an expected height for the protective mattress that would allow the calves and heels to sink enough without reaching the bottom of the mattress. More significantly, this strategy might complement a weakness that was present in a single-thickness layer : layers of different thicknesses are separated from each other so that the weight of the leg is sustained at each level of the layers while the loading due to the weight of the leg is gradually and intermittently distributed through the stratified layers ${ }^{7}$. One study indicated that heel skin perfusion increased with elevation $^{22)}$, and there is a risk of developing a pressure ulcer when the support pressure is greater than the capillary perfusion pressure ${ }^{5)}$. However, elevation of the legs in the present study ranged from only 34.2 to $49.2 \mathrm{~cm}$, which may not affect perfusion pressure, and the support pressure, or the peak pressure in the present study, was low.

Second, each layer was double-folded so that it would face the other layer with a 180-degree rotation. This strategy may maximize softness by stratifying the same kinds of materials while minimizing the actual amount of material used. The structure of each layer consisted of a middle part with the three dimensions sandwiched between different textures on the front and back. The back sides faced each other at the inside of the folded half, so that the contact space of the inside mat attached more smoothly than a space with two different kinds of texture. This characteristic may contribute to the softness of the doublefolded layer. The softness of the material was required to maintain the morphological shape of the calf and heel along with the lines of the contact area.

Finally, 90-degree rotation may minimize the weakness seen with stratified layers that were not rotated. The weight of the leg might have been sustained better by the 90 degree-rotated layers than by the non-rotated ones, because the 90-degree-rotated layers were stratified in a double-fold on top of another double-folded layer in order to support the vertical structure against horizontal displacement. Each 90degree-rotated layer may distribute the loading due to the weight of the leg, thus providing some firmness and both sustaining and preventing the leg from sinking to the bottom of the mattress. Such firmness might limit the expansion of the contact area more than that of non-rotated layers, but it did not limit the reduction of peak pressure.

The decreased deviation from the center point when the sliding motion on the mattresses was measured may indicate the effectiveness of 90-degree rotation. The leg may slide less if it is placed on a thick mattress with the stratified layers compared to that of just a single layer. In particular, the 90-degree-rotated, double-folded strategy may reduce the sliding motion. This strategy may allow the weight of the leg to further sink vertically downward, while firmly sustaining the leg's weight. Although there is no consensus on what level or type of shear is clinically relevant ${ }^{21)}$, decreasing the sliding motion as much as possible would be beneficial $^{7)}$. This, in turn, may decrease the shear force that causes skin damage and collapse of the capillary vessels over the contact areas.

Materials with constant low pressure devices or specification foam mattresses will firmly stabilize the weight of the body with a wider pressure distribution fitting body morphology ${ }^{23)}$. However, the low pressure devices such as low resilience mattress being recently manufactured by the 
urethane has the weakness of retaining distortion, and it takes time to return to a flat surface, so that patients have to keep shifting the position of their legs. On the other hand, our mattress with 3-dimensional soft knit fabric using stratification, double-folds, and 90-degree rotation may bring good resilience, returning quickly to a flat surface after the pressure of the weight is released.

There are some limitations to this study due to the use of a dummy human leg. Sensations, such as the sliding motion and support from the firmness when placed on the mattress could not be confirmed. In addition, changes in sensation, such as warmth due to decreased sympathetic nervous system activation from reduced peak pressure could not be determined.

In conclusion, the 3-D knit fabrics with 90-degree rotation allows the calves and heels to sink vertically downward while being gently held, and stabilized firmly without sinking to the mattress bottom. The mattresses developed by applying this framework of the present study may provide effective protection against skin and soft tissue damage from the interface pressure by increasing the contact area and from shear force by decreasing sliding motion. Protective mattresses might prevent severe blockage of the microcirculation of the legs, and, consequently, the signs and symptoms of PVD would be ameliorated by such mattresses when used by patients with PVD for rest and sleep.

\section{Funding}

This study was funded by an investigational grant from the University of Shizuoka. The outcome of this study was submitted to the Japan Patent Office to obtain a patent right in 2008 and, was disclosed in 2010.

\section{References}

1) Treiman, G. S., Copland, S., McNamara, R. M., Yellin, A. E., Schneider, P. A. \& Treiman, R. L. : Factors influencing ulcer healing inpatients with combined arterial and venous insufficiency. Journal of Vascular Surgery, 33 (6), 11581164, 2001.

2) Lyder, C. H. : Pressure ulcer prevention and management. The Journal of American Medical Association, 289 (2), 223226, 2003.

3) Defloor, T. \& Grypdonck, M. H. F. : Do pressure relief cushions really relieve pressure? Western Journal of Nursing Research, 22 (3), 335-350, 2000.

4) Mimura, M., Okazaki, H., Kajiwara, R., Ohura, T. \&
Takahashi, M. : Change of surface pressure and shear force during upper body raise/lower movements. [in Japanese with English abstract], Japanese Journal of Pressure Ulcer, 9 (1), 11-20, 2007.

5) Roeder, R. A., Geddes, L. A., Corson, N., Pell, C., Otlewski, M. \& Kemeny, A. : Heel and calf capillarysupport : Pressure in lithotomy positions. AORN Journal, $81(4), 821-830,2005$.

6) Wong, V. K. \& Stotts, N. A. : Physiology and prevention of heel ulcers : The state of science. Journal of Wound, Ostomy, and Continence Nursing, 30 (4) , 191-198, 2003.

7) Sakuta, Y. \& Takahashi, M. : The interaction between pressure and shear force as a factor of pressure ulcers by assessing blood flow measurement. [in Japanese with English abstract] Transactions of the Japanese Society for Medical and Biological Engineering, 44 (1), 101-106, 2006.

8) Landis, E. M. : Microinjection studies of capillary blood pressure in human skin. Heart, 15, 209-228, 1930.

9) Sugama, J., Sanada, H., Nakano, N., Masuya, N., Tabata, K., Kumakawa, R. \& Takahashi, M. : Reliability and validity of a new multi-pad pressure evaluator for pressure ulcer management. [ in Japanese with English abstract] Japanese Journal of Pressure Ulcer, 2 (3) , 310-315, 2000.

10) Girling, F. V.: Vasomotor effects of electrical stimulation. American Journal of Physiology, 170, 131-135, 1952.

11) Abraham, P., Fromy, B., Merzeau, S., Tardel, A. \& Saumet, J. L. : Dynamic of local pressure-induced cutaneous vasodilation in the human hand. Microvascular Research, 61 (1), 122-129, 2001.

12) Fromy, B., Abraham P., Bouvet, C., Bouhanick, B. Fressinaud, P. \& Saumet, J. L. : Early decrease of skin blood flow in response to locally applied pressure in diabetic subjects. Diabetes, 52, 1214-1217, 2002.

13) Burton, A. C. : Physiology and Biophysics of the Circulation. 2nd, Chicago : Year Book, 1972.

14) Sagawa, S., Ikeda, R., Higashi, T., Aso, Y., Ikenaga, H., Morito, N., Nishikawa, T., Yanagida, T. \& Seiyama, A. : Evaluation of anti-decubitus ulcer mattress : measuring blood volume of sacral region by NIRS. [in Japanese with English abstract], Journal of Japanese College of Angiology, 45, 101-105, 2005.

15) Reddy, M., Gill, S. S. \& Rochon, P. A. : Preventing pressure ulcers : A systematic review. The Journal of American Medical Association, 296 (8), 974-984, 2006.

16) Hemmes, B., Poeze, M. \& Brink, P. R. : Reduced tissueinterface pressure and increased comfort on a newly developed soft-layered long spineboad. Journal of Trauma, 68 (3), 593-598, 2010.

17) Gschwandtner, M. E., Ambrozy, E., Maric, S., Willfort, A. Schneider, B., Bohler, K., Gaqql, U. \& Ehringer, H. : Microcirculation is similar in ischemic and venous ulcers. Microvascular Research, 62 (3), 226-235, 2001.

18) Park, Se Jin, Kim, Chae-Bogk \& Park, Soo Chan : 
Anthropometric and biomechanical characteristics on body segments of Koreans. Applied Human Science, 18 (3), 9199, 1999.

19) Harada, C., Shigematsu, T. \& Hagisawa, S. : The effect of 10-degree leg elevation and 30-degree head elevation on body displacement and sacral interface pressure over a 2hour period. Journal of Wound, Ostomy, and Continence Nursing, 29, 143-148, 2002.

20）DeVocht, J. W., Wilder, D. G., Bandstra E. R. \& Spratt, K. F. : Biomechanical evaluation of four different mattresses. Applied Ergonomics, 37, 297-304, 2006.
21) Call, E. \& Baker, L. : How does bed frame design influence tissue interface pressure? A comparison of four different technologies designed for long-term or home care. Journal of Tissue Viability, 17, 22-29, 2007.

22) Huber, J., Reddv, R., Pitham, T. \& Huber, P. : Increasing heel skin perfusion by elevation. Advances in Skin \& Wound Care, 21 (1), 37-41, 2008.

23） Moysidis, T., Niebel, W., Bartsch, K., Maier, I., Lehmann, N. \& Nonnemacher, M., et al. : Prevention of pressure ulcer : interaction of body characteristics and different mattresses. International Wound Journal, 8 (6), 578-584, 2011. 